\title{
Associations between primary aldosteronism and diabetes, poor bone health, and sleep apnea-what do we know so far?
}

\author{
Huai Heng Loh $^{1} \cdot$ Norlela Sukor ${ }^{2}$
}

Received: 18 July 2019 / Revised: 12 November 2019 / Accepted: 2 December 2019 / Published online: 10 December 2019

(c) The Author(s), under exclusive licence to Springer Nature Limited 2019

\begin{abstract}
Primary aldosteronism (PA), the most common cause of secondary hypertension, is a well-recognized condition that can lead to cardiovascular and renal complications. PA is frequently left undiagnosed and untreated, leading to aldosterone-specific morbidity and mortality. In this review we highlight the evidence linking PA with other conditions such as (i) diabetes mellitus, (ii) obstructive sleep apnea, and (iii) bone health, along with clinical implications and proposed underlying mechanisms.
\end{abstract}

\section{Introduction}

Despite old beliefs that primary aldosteronism (PA) is uncommon and warrants screening only when patients present with hypertension and hypokalemia, it is now believed that PA is in fact the most common cause of secondary hypertension, accounting for $5-13 \%$ of the hypertensive cohort, with an even higher prevalence among those with resistant hypertension [1, 2]. This stems from more efficient screening of this condition with the widespread use of the aldosterone:renin ratio [3, 4]. It is a disorder in which the adrenal cortex autonomously produces excessive aldosterone leading to sodium and water retention, resulting in a subsequent rise of blood pressure (BP), and the downregulation of renin through the renin-angiotensin-aldosterone system (RAAS) pathway [5]. Aldosterone-producing adenoma (APA) and bilateral hyperaldosteronism (BHA) are the most common causes of PA, with the latter being more prevalent $[4,6]$. Unilateral adrenalectomy for APA can potentially cure and at least improves hypertension, and the mineralocorticoid receptor

Norlela Sukor

drlela2020@yahoo.com

1 Faculty of Medicine and Health Sciences, Universiti Malaysia Sarawak, Kota Samarahan, Sarawak, Malaysia

2 Faculty of Medicine, Department of Medicine, Universiti Kebangsaan Malaysia Medical Center, Kuala Lumpur, Malaysia antagonist (MRA) administration for BHA effectively controls hypertension in this condition [7, 8].

Once considered a relatively benign condition, PA has been unequivocally shown to have effects beyond its effects on BP levels $[9,10]$. Hypertension alone could not explain the higher adverse cardiovascular outcomes as PA patients were found to have a higher cardiovascular mortality compared with patients with essential hypertension (EH) despite being matched for cardiovascular risks [11]. PA-directed therapy was able to reduce the excess morbidity to a level comparable to subjects with $\mathrm{EH}$, regardless of the treatment modality [12, 13]. Subsequently, elevated aldosterone levels were shown to contribute to the excess morbidity and mortality [14]. Recent studies have shown that PA is associated with a myriad of other co-morbidities, particularly cardiovascular and renal injuries, which might play a role in contributing to the elevated morbidity and mortality [15]. What is less certain and less looked into is the association between PA and several nonclassical associations, namely diabetes, bone health, and sleep apnea.

Several large cardiovascular trials have suggested that the interruption of the RAAS prevented the occurrence of diabetes, raising the question of a link between PA and insulin resistance and diabetes. Besides the usual cardiovascular risk factors, obstructive sleep apnea (OSA) is known to contribute to the development of hypertension and plays an important role in resistant hypertension. There is high prevalence of OSA in resistant hypertension and it has been suggested that aldosterone is the hormone responsible for the resistant hypertension seen in this cohort of patients, 